\title{
TRANSMUTATIONS IN FUSION TEST FACILITIES
}

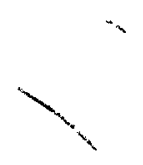

HEDL -7584

DE87 004069

\section{F.M. Mann \\ Date Published: April 1986}

\section{DISCLAIMER}

This report was prepared as an account of work sponsored by an agency of the United States Government. Neither the United States Government nor any agency thereof, nor any of their employees, makes any warranty, express or implied, or assumes any legal liability or responsibility for the accuracy, completeness, or usefulness of any information, apparatus, product, or proces the its use would not infringe privately owned rights. Referprocess discosed secific commercial product, process, or service by trade name, trademark, ence herein to any specific commen not necessarily constitute or imply its endorsement, recommanufacturer, or otherwise does not necessarily constite or imply its end thereof. The views mendation, or favoring by the United States Government or any agency the those of the and opinions of authors expressed herein do not necessarily state or reflect those of the United States Government or any agency thereof. 


\section{DISCLAIMER}

Portions of this document may be illegible in electronic image products. Images are produced from the best available original document. 


\section{TRANSMUTATIONS IN FUSION TEST FACILITIES}

F. M. Mann (Hanford Engineering Development Laboratory)

$1.0 \quad$ Objective

The objective of this work is to determine how typical materials transmute in various fusion test facilities.

$2.0 \quad$ Summary

Using an expanded nuclear data base, the transmutation of PCA, AMCR33 (a reduced activation austenitic stee1), HT-9, Rafer2 (a reduced activation ferritic steel), $V-15 \% \mathrm{Cr}-5 \% \mathrm{~T} i$ alloy, and SiATON (a ceramic) were calculated for two positions in the Fast Flux Test Facility (FFTF), three positions in the High Flux Isotope Reactor (HFIR), and the first wall position of both the STARFIRE and MARS conceptual fusion reactors. The peripheral test (PTP) position, and to a lesser extent the radial beryllium (RB) position, of HFIR show significant transmutations which are often in the opposite direction to the transmutations in the fusion conceptual designs. The positions in FFTF, as well as the hafnium covered location in the HFIR RB position show relative minor transmutations.

\section{$3.0 \quad$ Program}

Title: Irradiation Effects Analysis Principal Investigator: D. G. Doran Affiliation: Westinghouse Hanford Company

\subsection{Relevant Program Plan Task/Subtask}

II.A.4 Gas Generation Rates

II.C. 4 and II.C.10 Effects of Solid Transmutants

\subsection{Accomplishments and Status}

\section{$5.1 \quad$ Introduction}

Because no facility exists which has a spectrum similar to that expected in a fusion reactor, existing fission reactors are being used to test the behavior of materials. Although it is believed that the change in material behavior will be most sensitive to displacement damage, the transmutation of composition has long been considered important. The major effort is in the gaseous transmutation products, particularly helium. However, now the importance of solid transmutants is being raised. Also, it is becoming realized that the transmutations in thermal reactors can be very large and can completely distort the composition of a material.

To determine the compositional change in typical fusion materials, calculations were performed for the first wall positions of two conceptual fusion designs and for positions in high flux test reactors used by the fusion materials program. The materials were chosen to be candidate first wall materials an insulator, and various elements to provide a broad treatment of possible material combinations. first wall candidates, reduced activation versions were also included. 
To gain insight into the behavior in a fusion facility, the first wall positions of the STARFIRE and MARS conceptual designs were chosen. Because of its use of water as a coolant, STARFIRE has a strong $1 / E$ low energy neutron flux shape. The MARS design, on the other hand, has relatively few low energy neutrons. Most of the high fluence irradiations for the fusion materials program will be in the FFTF or the HFIR. Therefore, two positions were chosen in the FFTF, one at core center [comparable to the midplane (or highest flux) position of the Material Open Test Assembly (MOTA)], and the other at the midplane position of the outer row (which is similar to the top of the MOTA). Calculations for three positions in HFIR were also performed, the first corresponding to the PTP, the second to a radial beryllium (RB) position, and the last to a hafnium shielded radial beryllium (RB-Hf) position. The fluxes for the last two positions (RB and RB-Hf) were kindly supplied by L. Greenwood (Argonne National Laboratory) from his dosimetry measurements, while fluxes for the other positions were taken from the standard REAC flux library.

The cross section and decay data needed for the calculations were taken from the newly expanded REAC libraries. (1) It should be noted that for many of the reactions, the needed cross sections were estimated.

The REAC computer code system(2) was used to calculate the change in composition for a six-year exposure at each position. For the HFIR positions, time steps were taken to be 0.125 year; for the other positions time steps were taken to be 0.25 year. The materials included in the calculation are PCA, VCrTi, HT-9, AMCR33 (a reduced activation austenitic), RAFER2 (a reduced activation ferritic), CuBe, Sialon, $\mathrm{Be}, \mathrm{C}, \mathrm{Al}, \mathrm{Si}, \mathrm{Cu}, \mathrm{Mo}, \mathrm{Nb}$, and $\mathrm{W}$.

\subsection{Results of the Calculations}

Tables 1 through 15 give the results of the calculations for an exposure of five years. Figures 1 and 2 show the change of $V$ in HT-9 and the change of Mn in Rafer2, respectively, as a function of exposure.

In general, the most significant transmutations occur for the PTP position of HFIR, particularly for odd mass and for high mass nuclei. The RB positions of HFIR often show similar but sinaller changes than the PTP positions. The hafnium-covered RB position (1ike the FFIF positions) shows practically no change in composition. The first wall positions show little change. The most notable changes in composition at a five-year exposure are: (1) the large burnout of Mn in HFIR (for PTP and RB) contrasted to the increase shown for STARFIRE and MARS for PCA, (2) the switch in composition from $80 \% \mathrm{~V} 15 \% \mathrm{Cr} 5 \% \mathrm{Ti}$ to $12 \% \mathrm{~V}$ 67\%Cr $5 \% \mathrm{~T}$ again at the PTP position, (3) the $1300 \%$ increase in iron in VCrTi for PTP, (4) the $200 \%$ increase of Mn in HT-9 in the fusion designs, (5) the burnout of Mn (which replaces $N i$ in the reduced austenitic AMCR33) in PTP from an initial 15\% to $1.5 \%$, and (6) the transformation of $N$ (initially $36 \%$ ) to $C$ (ending will be 18\%) for Sialon in PTP. Various new elements are also created. How significant these additions are depends upon their metallurgical importance.

It should be noted some of the compositional changes are not linear functions of time. Vanadium in HT-9 (see Figure 1) shows this quite dramatically for the PTP position in HFIR. The amount of $V$ first increases (as it does for all the positions studied), but then in the PTP position the vanadium burns out.

$5.7 \quad$ Conclusion

Whenever materials are exposed to a significant fluence in the PTP position in HFIR, the compositional change of the material should be investigated. However, other facilities (including fusion devices) also show some large changes for certain materials.

\section{$6.0 \quad$ References}

1. F. M. Mann, "Cross Sections for Reduced Activation Studies", DAFS Quarterly Progress Report, DOE/ER-0046/20, February 1985, P. 18 .

2. F. M. Mann, "Transmutation of Alloys in MFE Facilities as Calculated by REAC (A Computer Code System for Activation and Transmutation", HEDL-TME-81-37, Westinghouse Hanford Company. 


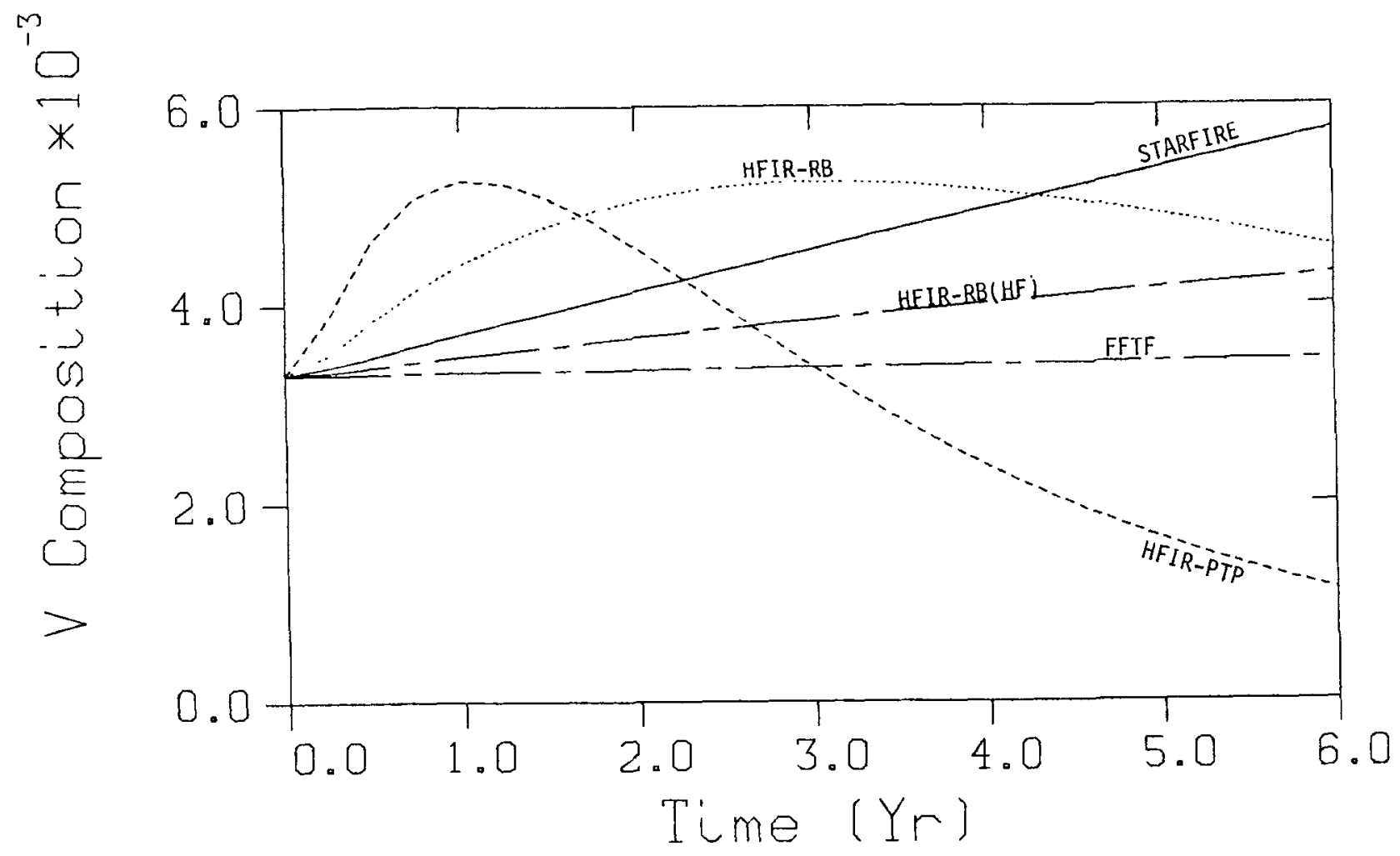

FIGURE 1. The Amount of $V$ in HT-9 as a Function of Time in STARFIRE, FFTF, and Three Positions in HFIR.

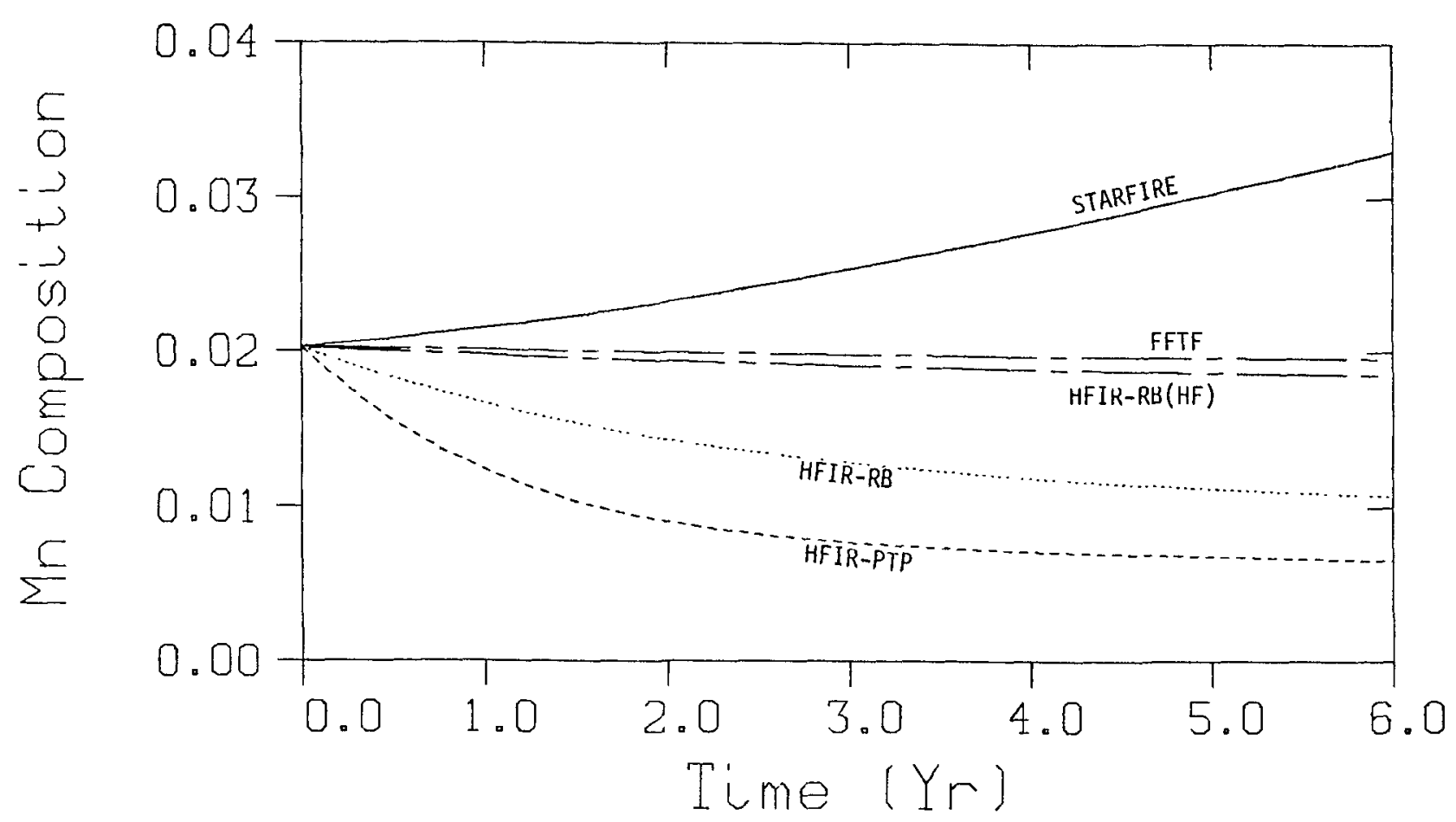

FIGURE 2. The Amount of $M n$ in RAFER2 as a function of time in STARFIRE, FFTF, and Three Positions in HFIR. 
TABLE 1

TRANSMUTATIONS IN PCA AFTER 5 YEARS IN 7 FUSION TEST FACILITIES

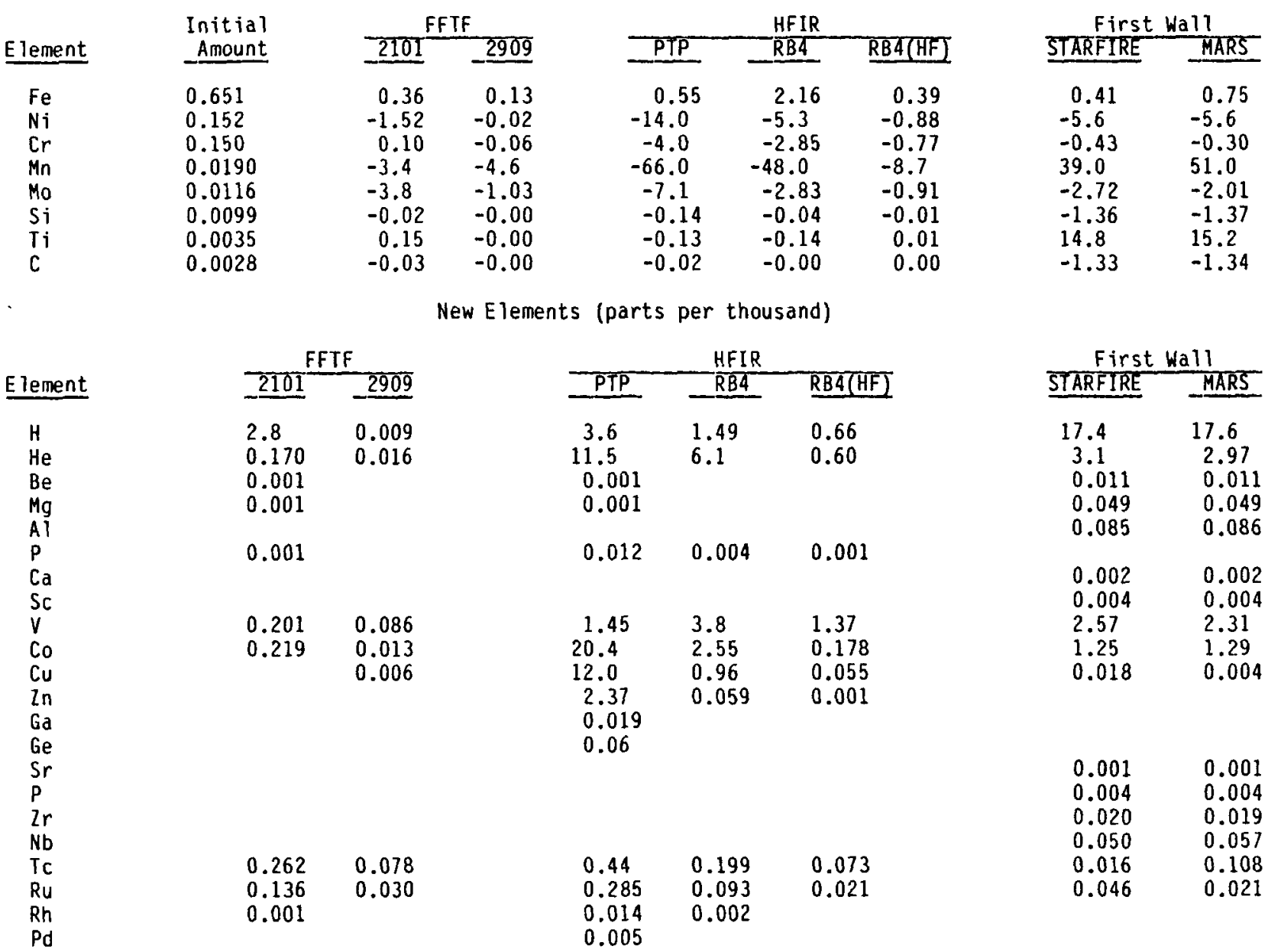

TABLE 2

TRANSMUTATIONS IN VCrTi AFTER 5 YEARS IN 7 FUSION TEST FACILITIES

\begin{tabular}{|c|c|c|c|c|c|c|c|c|}
\hline \multirow[b]{3}{*}{ Element } & \multirow{3}{*}{$\begin{array}{r}\text { Initial } \\
\text { Amount } \\
\end{array}$} & \multicolumn{5}{|c|}{ Fractional Change $(\%)$ in Initial Constituents } & \multirow{2}{*}{\multicolumn{2}{|c|}{ First Wall }} \\
\hline & & \multicolumn{2}{|c|}{ FFTF } & \multicolumn{3}{|c|}{ HFIR } & & \\
\hline & & 2101 & 2909 & PTP & RB4 & RB4(HF) & STARFIRE & MARS \\
\hline $\begin{array}{l}\mathrm{V} \\
\mathrm{Cr} \\
\mathrm{Ti} \\
\mathrm{N} \\
0 \\
\mathrm{C} \\
\mathrm{Fe} \\
\mathrm{Mo} \\
\mathrm{Cu}\end{array}$ & $\begin{array}{l}0.797 \\
0.146 \\
0.0528 \\
0.00189 \\
0.00073 \\
0.00072 \\
0.00050 \\
0.00030 \\
0.00005\end{array}$ & $\begin{array}{c}-1.22 \\
6.6 \\
0.07 \\
2.66 \\
-0.13 \\
3.7 \\
-0.10 \\
-0.38 \\
-5.8\end{array}$ & $\begin{array}{r}-0.50 \\
2.70 \\
-0.00 \\
-0.17 \\
-0.00 \\
0.44 \\
-0.02 \\
-1.03 \\
-2.07\end{array}$ & $\begin{array}{c}-85.0 \\
460.0 \\
-1.28 \\
-50.0 \\
-0.15 \\
128.0 \\
1270.0 \\
-7.1 \\
-66.0\end{array}$ & $\begin{array}{l}-46.0 \\
248.0 \\
-0.17 \\
-20.0 \\
-0.03 \\
52.0 \\
36.0 \\
-2.82 \\
-36.0\end{array}$ & $\begin{array}{l}-7.6 \\
41.0 \\
-0.01 \\
-3.2 \\
-0.03 \\
7.8 \\
0.14 \\
0.91 \\
-6.4\end{array}$ & $\begin{array}{l}-2.20 \\
9.3 \\
7.1 \\
-2.98 \\
-1.33 \\
5.0 \\
-1.71 \\
-2.73 \\
-7.6\end{array}$ & $\begin{array}{l}-0.40 \\
-0.51 \\
7.2 \\
-2.81 \\
-1.32 \\
4.3 \\
-1.80 \\
-2.01 \\
-4.9\end{array}$ \\
\hline
\end{tabular}


TABLE 2 (Cont'd)

New Elements (parts per thousand)

\begin{tabular}{l} 
Element \\
\hline$H$ \\
$H e$ \\
$\mathrm{He}$ \\
$\mathrm{Be}$ \\
$\mathrm{B}$ \\
$\mathrm{Ca}$ \\
$\mathrm{SC}$ \\
$\mathrm{Mn}$ \\
$\mathrm{Co}$ \\
$\mathrm{Ni}$ \\
$\mathrm{Zn}$ \\
$\mathrm{Zr}$ \\
$\mathrm{Nb}$ \\
$\mathrm{TC}$ \\
$\mathrm{Ru}$
\end{tabular}

\begin{tabular}{ll}
\multicolumn{2}{c}{$F F T F$} \\
\hline 2101 & -2909 \\
0.174 & 0.004 \\
0.037 & \\
& \\
0.025 & \\
0.001 & \\
0.001 & \\
0.006 & 0.001 \\
0.001 & 0.001 \\
0.001 & \\
& \\
0.007 & 0.002 \\
0.004 & 0.001
\end{tabular}

\begin{tabular}{|c|c|c|}
\hline \multicolumn{3}{|c|}{ HFIR } \\
\hline PTP & RB4 & RB4(HF) \\
\hline $\begin{array}{l}1.07 \\
0.035\end{array}$ & $\begin{array}{l}0.40 \\
0.009\end{array}$ & $\begin{array}{l}0.083 \\
0.007\end{array}$ \\
\hline $\begin{array}{l}0.020 \\
0.001\end{array}$ & 0.005 & 0.005 \\
\hline $\begin{array}{l}7.6 \\
0.023 \\
0.018 \\
0.008\end{array}$ & $\begin{array}{l}0.55 \\
0.001 \\
0.009 \\
0.003\end{array}$ & $\begin{array}{l}0.021 \\
0.002\end{array}$ \\
\hline $\begin{array}{l}0.011 \\
0.007\end{array}$ & $\begin{array}{l}0.005 \\
0.002\end{array}$ & $\begin{array}{l}0.002 \\
0.001\end{array}$ \\
\hline
\end{tabular}

First WaI STARFIRE

$\begin{array}{ll}5.3 & 5.3 \\ 1.32 & 1.33 \\ 0.003 & 0.003 \\ 0.003 & 0.003 \\ 0.017 & 0.019 \\ 0.033 & 0.033 \\ 0.065 & 0.067 \\ 0.012 & 0.009 \\ 0.003 & 0.002 \\ 0.001 & \\ 0.001 & 0.001 \\ 0.004 & 0.003 \\ 0.001 & 0.001\end{array}$

TABLE 3

TRANSMUTATIONS IN HT-9 AFTER 5 YEARS IN 7 FUSION FACILITIES

Fractional Change (\%) in Initial Constituents

\begin{tabular}{lll} 
Element & & $\begin{array}{c}\text { Initial } \\
\text { Amount }\end{array}$ \\
\cline { 1 - 1 } $\mathrm{Fe}$ & & 0.840 \\
$\mathrm{Cr}$ & & 0.122 \\
$\mathrm{C}$ & & 0.0092 \\
$\mathrm{Si}$ & & 0.0078 \\
$\mathrm{Mo}$ & & 0.0057 \\
$\mathrm{Mn}$ & & 0.0055 \\
$\mathrm{Ni}$ & & 0.0047 \\
$\mathrm{~V}$ & & 0.0033 \\
$\mathrm{~W}$ & & 0.0015 \\
$\mathrm{P}$ & & 0.0003 \\
$\mathrm{~S}$ & & 0.0003
\end{tabular}

\begin{tabular}{cc}
\multicolumn{2}{c}{ FFTF } \\
\hline 2101 & -2909 \\
-0.09 & 0.01 \\
0.27 & -0.04 \\
-0.03 & -0.00 \\
0.03 & 0.00 \\
-3.8 & -1.03 \\
5.2 & -2.00 \\
-1.52 & -0.01 \\
3.8 & 1.62 \\
-17.5 & -29.2 \\
-0.35 & -0.03 \\
-0.67 & 0.03
\end{tabular}

\begin{tabular}{ccc} 
& \multicolumn{3}{c}{$H F I R$} \\
\hline PTP & RB4 & RB4 \\
\cline { 3 - 4 }-3.5 & -0.34 & -0.04 \\
-1.68 & -1.58 & -0.64 \\
-0.02 & -0.00 & -0.00 \\
-0.07 & -0.03 & 0.00 \\
-7.1 & -2.82 & 4.7 \\
14.9 & 14.6 & -0.90 \\
154.0 & 1.70 & -0.71 \\
-50.0 & 49.0 & 26.0 \\
-90.0 & -59.0 & -35.0 \\
-3.9 & -1.31 & -0.21 \\
5.1 & 1.93 & 0.13
\end{tabular}

New Elements (parts per thousand)

\begin{tabular}{l} 
Element \\
\hline$H$ \\
$\mathrm{He}$ \\
$\mathrm{Be}$ \\
$\mathrm{Mg}$ \\
$\mathrm{Al}$ \\
$\mathrm{Cl}$ \\
$\mathrm{Ti}$ \\
$\mathrm{Co}$ \\
$\mathrm{Cu}$ \\
$\mathrm{Zn}$ \\
$\mathrm{Ga}$ \\
$\mathrm{Sr}$ \\
$\mathrm{Y}$ \\
$\mathrm{Zr}$ \\
$\mathrm{Nb}$ \\
$\mathrm{TC}$ \\
$\mathrm{Ru}$
\end{tabular}

\begin{tabular}{ll}
\multicolumn{2}{c}{ FFTF } \\
\hline 2101 & $-\frac{2909}{200}$ \\
0.85 & 0.002 \\
0.078 & 0.001 \\
0.002 & \\
0.001 & \\
& \\
0.005 & \\
0.035 & \\
0.001 & \\
& \\
& \\
& \\
& \\
0.129 & 0.038 \\
0.067 & 0.015
\end{tabular}

\begin{tabular}{ccc}
\multicolumn{3}{c}{ HFIR } \\
\hline PTP & RB4 & RB4(HF) \\
\cline { 1 - 1 } 0.75 & 0.189 & 0.171 \\
0.42 & 0.206 & 0.032 \\
0.002 & & \\
0.001 & & \\
0.001 & & \\
0.007 & 0.001 & 0.001 \\
24.6 & 2.27 & 0.056 \\
0.493 & 0.030 & 0.002 \\
0.083 & 0.002 & \\
0.001 & & \\
& & \\
& & \\
0.214 & 0.098 & 0.036 \\
0.140 & 0.046 & 0.010
\end{tabular}

First Wall

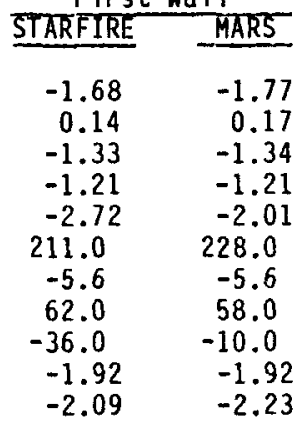

First Wall

\begin{tabular}{lc} 
STARFIRE & MARS \\
\cline { 1 - 1 } 12.3 & \\
2.33 & 12.4 \\
0.036 & 2.34 \\
0.039 & 0.036 \\
0.067 & 0.039 \\
& 0.068 \\
0.44 & \\
0.065 & 0.45 \\
0.001 & 0.045 \\
& \\
& \\
0.001 & \\
0.002 & 0.001 \\
0.010 & 0.002 \\
0.025 & 0.010 \\
0.080 & 0.028 \\
0.023 & 0.053 \\
& 0.010
\end{tabular}


TABLE 3 (Cont'd)

New Elements (parts per thousand)

\begin{tabular}{l} 
Element \\
\hline $\mathrm{Rh}$ \\
$\mathrm{Pd}$ \\
$\mathrm{Ta}$ \\
$\mathrm{Re}$ \\
$\mathrm{Os}$ \\
$\mathrm{Ir}$ \\
$\mathrm{Pt}$ \\
$\mathrm{Au}$ \\
$\mathrm{Hg}$ \\
$\mathrm{Tl}$ \\
$\mathrm{Pb}$
\end{tabular}

\begin{tabular}{ll}
\multicolumn{2}{c}{ FFTF } \\
\hline$\underline{2101}$ & -2909 \\
0.001 & \\
0.001 & 0.001 \\
0.176 & 0.233 \\
0.083 & 0.183 \\
& 0.016 \\
& 0.002 \\
&
\end{tabular}

\begin{tabular}{|c|c|c|}
\hline PTP & $-\frac{\mathrm{HFIR}}{\mathrm{RB4}}$ & RB4(HF \\
\hline $\begin{array}{l}0.007 \\
0.003\end{array}$ & 0.001 & \\
\hline $\begin{array}{l}0.118 \\
0.65 \\
0.079\end{array}$ & $\begin{array}{l}0.233 \\
0.119 \\
0.278 \\
0.112 \\
0.006\end{array}$ & $\begin{array}{l}0.111 \\
0.191 \\
0.167 \\
0.045\end{array}$ \\
\hline $\begin{array}{l}0.33 \\
0.37 \\
0.059\end{array}$ & $\begin{array}{l}0.109 \\
0.003\end{array}$ & \\
\hline
\end{tabular}

First Wall

STARFIRE MARS

0.001

$0.003 \quad 0.033$

$0.210 \quad 0.108$

$0.241 \quad 0.008$

0.066

0.015

TABLE 4

TRANSMUTATIONS IN AMCR33 AFTER 5 YEARS IN 7 FUSION TEST FACILITIES AMCRSS IS A REDUCED ACTIVATION AUSTENITIC ALLOY

Fractional Change (\%) in Initial Constituents

\begin{tabular}{|c|c|c|c|c|c|c|}
\hline \multirow[b]{2}{*}{ Element } & \multirow{2}{*}{$\begin{array}{c}\text { Initial } \\
\text { Amount }\end{array}$} & \multicolumn{2}{|c|}{ FFTF } & \multicolumn{3}{|c|}{ HFIR } \\
\hline & & 2101 & 2909 & PTP & RB4 & RB4(HF) \\
\hline $\begin{array}{l}\mathrm{Fe} \\
\mathrm{Mn} \\
\mathrm{Cr} \\
\mathrm{Si} \\
\mathrm{C} \\
\mathrm{Ni}\end{array}$ & $\begin{array}{l}0.723 \\
0.155 \\
0.107 \\
0.0107 \\
0.0048 \\
0.0005\end{array}$ & $\begin{array}{l}1.18 \\
-5.8 \\
0.24 \\
-0.02 \\
-0.03 \\
-1.49\end{array}$ & $\begin{array}{l}1.12 \\
-5.2 \\
-0.06 \\
-0.00 \\
-0.00 \\
0.05\end{array}$ & $\begin{array}{r}15.7 \\
-91.0 \\
-3.9 \\
-0.01 \\
-0.02 \\
1480.0\end{array}$ & $\begin{array}{l}13.5 \\
-65.0 \\
-2.83 \\
-0.04 \\
-0.00 \\
53.0\end{array}$ & $\begin{array}{c}2.59 \\
-12.1 \\
-0.85 \\
-0.01 \\
-0.00 \\
-0.29\end{array}$ \\
\hline
\end{tabular}

New Elements (parts per thousand)

\begin{tabular}{|c|c|c|c|c|c|c|c|}
\hline \multirow[b]{2}{*}{ Element } & \multicolumn{2}{|c|}{ FFTF } & \multicolumn{3}{|c|}{ HFIR } & \multicolumn{2}{|c|}{ First Wall } \\
\hline & 2101 & 2909 & PTP & RB4 & RB4(HF) & START FIRE & MARS \\
\hline $\begin{array}{l}\mathrm{H} \\
\mathrm{He} \\
\mathrm{Be} \\
\mathrm{Mg} \\
\mathrm{Al}\end{array}$ & $\begin{array}{l}0.69 \\
0.065 \\
0.001 \\
0.001\end{array}$ & 0.001 & $\begin{array}{l}0.60 \\
0.105 \\
0.001 \\
0.001\end{array}$ & $\begin{array}{l}0.135 \\
0.035\end{array}$ & $\begin{array}{l}0.138 \\
0.014\end{array}$ & $\begin{array}{l}10.9 \\
2.12 \\
0.018 \\
0.053 \\
0.093\end{array}$ & $\begin{array}{c}11.0 \\
2.14 \\
0.019 \\
0.053 \\
0.093\end{array}$ \\
\hline $\begin{array}{l}\mathrm{P} \\
\mathrm{Ti} \\
\mathrm{V} \\
\mathrm{Co} \\
\mathrm{Cu} \\
\mathrm{Zn}\end{array}$ & $\begin{array}{l}0.001 \\
0.004 \\
0.145 \\
0.024\end{array}$ & $\begin{array}{l}0.061 \\
0.010\end{array}$ & $\begin{array}{c}0.013 \\
0.006 \\
1.01 \\
23.3 \\
0.150 \\
0.017\end{array}$ & $\begin{array}{l}0.004 \\
0.001 \\
2.73 \\
2.01 \\
0.003\end{array}$ & $\begin{array}{l}0.001 \\
0.001 \\
0.98 \\
0.043\end{array}$ & $\begin{array}{l}0.38 \\
1.88 \\
0.026\end{array}$ & $\begin{array}{l}0.38 \\
1.70 \\
0.008\end{array}$ \\
\hline
\end{tabular}

First Wall

\begin{tabular}{cc} 
STARFIRE & MARS \\
\hline 0.48 & -1.70 \\
-6.1 & 3.4 \\
3.5 & 3.8 \\
-1.37 & -1.37 \\
-1.34 & -1.35 \\
-5.3 & -5.6
\end{tabular}

First Wall

\begin{tabular}{l|l}
10.9 & 11.0 \\
2.12 & 2.14 \\
0.018 & 0.019 \\
0.053 & 0.053 \\
0.093 & 0.093 \\
& \\
0.38 & 0.38 \\
1.88 & 1.70 \\
0.026 & 0.008
\end{tabular}


TABLE 5

TRANSMUTATIONS IN RAFER2 AFTER 5 YEARS IN 7 FUSION TEST FACILITIES RAFER2 IS A REDUCEO ACTIVATION FERRITIC ALLOY

Fractional Change (\%) in Initial Constituents

$\begin{array}{lll}\text { Element } & & \begin{array}{l}\text { Initial } \\ \text { Amount }\end{array} \\ \mathrm{Fe} & & 0.863 \\ \mathrm{Cr} & & 0.0961 \\ \mathrm{Mn} & & 0.0203 \\ \mathrm{~W} & & 0.01305 \\ \mathrm{C} & & 0.00457\end{array}$

\begin{tabular}{cc}
\multicolumn{2}{c}{ FFTF } \\
\hline$-\frac{2101}{2}$ & -2909 \\
0.01 & 0.10 \\
-3.0 & -0.00 \\
0.40 & -4.4 \\
-17.5 & -29.2 \\
-0.03 & -0.00
\end{tabular}

\begin{tabular}{|c|c|c|}
\hline & HFIR & \\
\hline PTP & RB4 & RB4 (HF) \\
\hline $\begin{array}{l}-2.05 \\
-1.46 \\
-66.0 \\
-90.0 \\
-0.02\end{array}$ & $\begin{array}{r}0.77 \\
-1.48 \\
-45.0 \\
-59.0 \\
-0.00\end{array}$ & $\begin{array}{c}0.17 \\
-0.60 \\
-7.7 \\
-35.0 \\
-0.00\end{array}$ \\
\hline
\end{tabular}

New Elements (parts per thousand)

Element

\begin{tabular}{ll}
\multicolumn{2}{c}{ FFTF } \\
\hline-2101 & $-\underline{2909}$ \\
0.78 & 0.001 \\
0.070 & \\
0.001 & \\
0.004 & \\
0.028 & 0.012
\end{tabular}

\begin{tabular}{lll}
\multicolumn{3}{c}{ HFIR } \\
\hline PTP & RB4 & RB4(HFI \\
\cline { 1 - 1 } 0.66 & 0.147 & 0.155 \\
0.069 & 0.016 & 0.013 \\
0.001 & & \\
0.005 & 0.001 & 0.001 \\
25.4 & 2.31 & 0.051 \\
8.4 & 0.342 & 0.003 \\
0.127 & & \\
0.010 & &
\end{tabular}

$\begin{array}{ll}0.005 & 0.007 \\ 1.53 & 1.59 \\ 0.72 & 0.180 \\ & 0.139 \\ & 0.017\end{array}$

0.010

TABLE 6

TRANSMUTATIONS IN CUBE AFTER 5 YEARS IN 7 FUSION TEST FACILITIES

$\begin{array}{ll}\text { Element } & \begin{array}{c}\text { Initial } \\ \text { Amount }\end{array} \\ & 0.874 \\ \mathrm{Be} & 0.126\end{array}$

Fractional Change $(\tilde{z})$ in Initial Constituents

\begin{tabular}{llllll}
\multicolumn{2}{c}{ FFTF } & \multicolumn{3}{c}{ HFIR } \\
\cline { 6 - 7 } 2101 & -2909 & & PTP & - RB4 & RB4(HF) \\
-4.4 & -1.38 & & -43.0 & -23.6 & -4.2 \\
-2.26 & -0.01 & & -2.66 & -0.48 & -0.46
\end{tabular}

New Elements (parts per thousand)

\begin{tabular}{l} 
Element \\
\hline $\mathrm{H}$ \\
$\mathrm{He}$ \\
$\mathrm{Li}$ \\
$\mathrm{Fe}$ \\
$\mathrm{Co}$ \\
$\mathrm{Ni}$ \\
$\mathrm{Zn}$ \\
$\mathrm{Ga}$ \\
$\mathrm{Ge}$
\end{tabular}

\begin{tabular}{|c|c|c|c|c|}
\hline \multicolumn{2}{|c|}{ FFTF } & \multicolumn{3}{|c|}{ HFIR } \\
\hline 2101 & 2909 & PTP & RB4 & RB4(HF) \\
\hline $\begin{array}{l}2.96 \\
3.40 \\
0.46\end{array}$ & $\begin{array}{l}0.007 \\
0.008 \\
0.003\end{array}$ & $302 .(?)$ & $\begin{array}{l}1.34 \\
0.49\end{array}$ & $\begin{array}{l}0.49 \\
0.77 \\
0.011\end{array}$ \\
\hline $\begin{array}{c}0.077 \\
25.7 \\
9.06\end{array}$ & $\begin{array}{l}8.9 \\
3.2\end{array}$ & $\begin{array}{c}0.216 \\
219.0 \\
148.0 \\
5.3 \\
2.92\end{array}$ & $\begin{array}{c}0.010 \\
151.0 \\
56.0 \\
0.197 \\
0.033\end{array}$ & $\begin{array}{c}0.015 \\
28.3 \\
8.6\end{array}$ \\
\hline
\end{tabular}

First Wall

\begin{tabular}{|c|c|}
\hline STARFIRE & MARS \\
\hline $\begin{array}{c}-1.53 \\
1.16 \\
50.0 \\
-36.0 \\
-1.33\end{array}$ & $\begin{array}{c}-1.79 \\
1.18 \\
61.0 \\
-10.0 \\
-1.35\end{array}$ \\
\hline
\end{tabular}

First Wall

\begin{tabular}{|c|c|}
\hline STARFIRE & MARS \\
\hline $\begin{array}{l}12.0 \\
2.08 \\
0.018 \\
0.35 \\
0.026 \\
0.002\end{array}$ & $\begin{array}{l}12.1 \\
2.09 \\
0.018 \\
0.36 \\
0.005\end{array}$ \\
\hline $\begin{array}{l}0.003 \\
0.022 \\
1.82 \\
2.10 \\
0.58 \\
0.134\end{array}$ & $\begin{array}{l}0.005 \\
0.287 \\
0.94 \\
0.068\end{array}$ \\
\hline
\end{tabular}

$\begin{array}{lll} & 0.055 & 0.003 \\ 2.87 & 0.95 & 0.001 \\ 3.2 & 0.030 & \\ 0.52 & & \end{array}$

First Wall

\begin{tabular}{ll}
\multicolumn{2}{c}{ First Wall } \\
${ } }$ & MARS \\
\hline-5.9 & -4.1 \\
-3.8 & -3.9
\end{tabular}

First Wall

\begin{tabular}{|c|c|}
\hline STAR FIRE & MARS \\
\hline $\begin{array}{c}10.4 \\
8.5 \\
0.197 \\
0.019 \\
1.97 \\
44.0 \\
5.4\end{array}$ & $\begin{array}{c}10.2 \\
8.5 \\
0.47 \\
0.021 \\
2.05 \\
32.0 \\
1.70\end{array}$ \\
\hline
\end{tabular}


TABLE 7

TRANSMUTATIONS IN SIALON AFTER 5 YEARS IN 7 FUSION TEST FACILITIES

Fractional Change (\%) in Initial Constituents

\begin{tabular}{|c|c|c|c|c|c|c|c|c|}
\hline \multirow[b]{2}{*}{ Element } & \multirow{2}{*}{$\begin{array}{l}\text { Initial } \\
\text { Amount }\end{array}$} & \multicolumn{2}{|c|}{ FFTF } & \multicolumn{3}{|c|}{ HFIR } & \multicolumn{2}{|c|}{ First Wa 11} \\
\hline & & 2101 & 2909 & PTP & RB4 & RB4(HFI & STARFIRE & MARS \\
\hline $\begin{array}{l}N \\
0 \\
A ! \\
S i\end{array}$ & $\begin{array}{l}0.357 \\
0.214 \\
0.214 \\
0.214\end{array}$ & $\begin{array}{r}-2.66 \\
-0.13 \\
-0.28 \\
0.24\end{array}$ & $\begin{array}{r}-0.17 \\
-0.00 \\
-0.04 \\
0.04\end{array}$ & $\begin{array}{c}-50.0 \\
-0.16 \\
-8.4 \\
8.2\end{array}$ & $\begin{array}{r}-20.0 \\
-0.03 \\
-2.81 \\
2.76\end{array}$ & $\begin{array}{r}-32.0 \\
-0.03 \\
-0.39 \\
0.38\end{array}$ & $\begin{array}{l}-2.96 \\
-1.33 \\
-0.46 \\
-1.24\end{array}$ & $\begin{array}{l}-2.78 \\
-1.32 \\
-0.39 \\
-1.31\end{array}$ \\
\hline
\end{tabular}

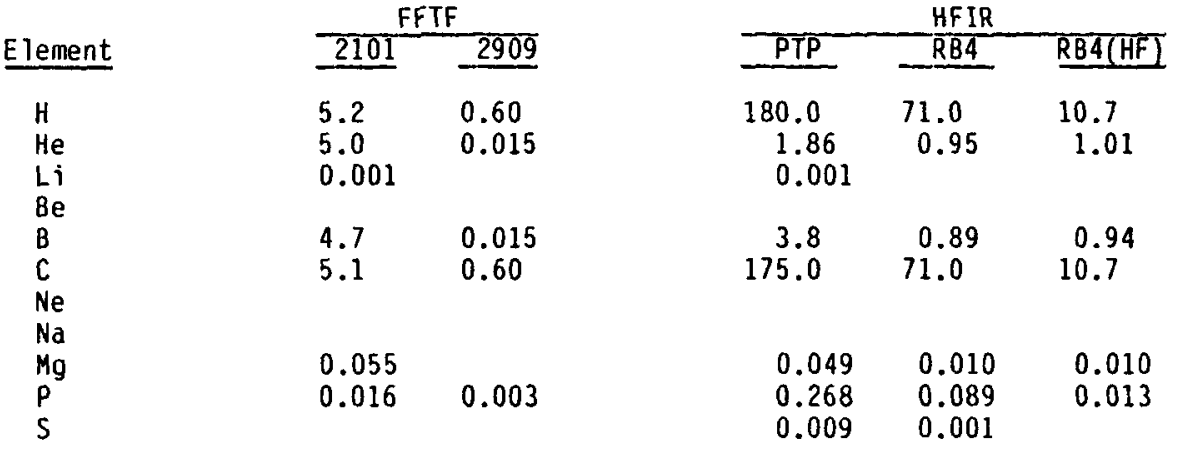

First Wall

\begin{tabular}{ll}
\multicolumn{1}{c}{ STARFIRE } & MARS \\
\cline { 1 - 1 } 14.1 & \\
9.5 & 13.1 \\
0.56 & 9.8 \\
0.024 & 0.53 \\
3.3 & 0.026 \\
9.5 & 3.6 \\
0.004 & 8.6 \\
0.024 & 0.005 \\
3.6 & 0.024 \\
0.006 & 3.6 \\
& 0.003
\end{tabular}

TABLE 8

TRANSMUTATIONS IN BE AFTER 5 YEARS IN 7 FUSION TEST FACILITIES

Fractional Change (\%) in Initial Constituents

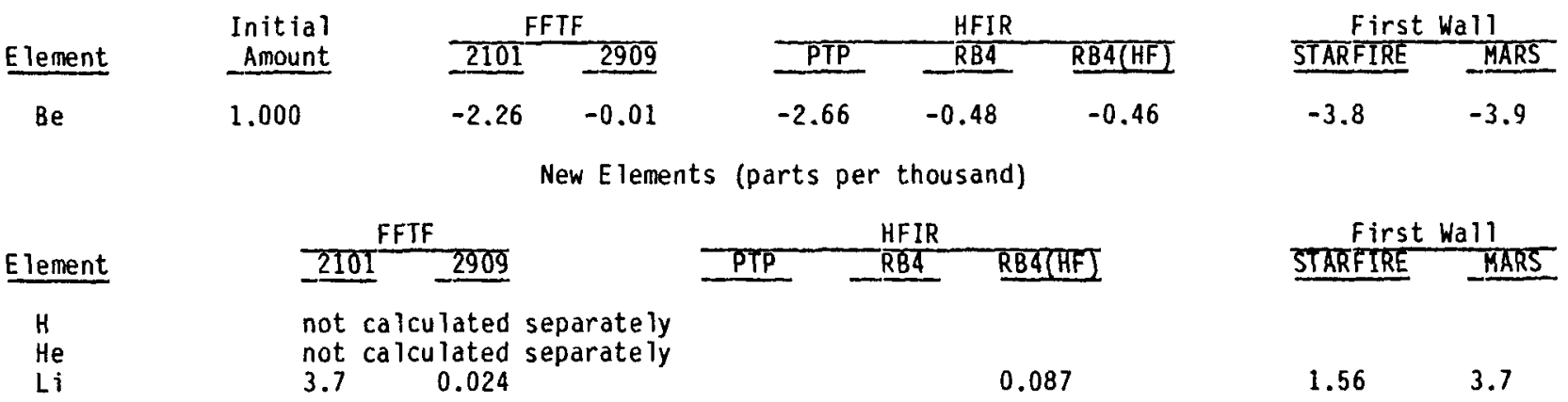

TABLE 9

TRANSMUTATION IN C AFTER 5 YEARS IN 7 FUSION TEST FACILITIES

Fractional Change (q) in Initial Constituents

\begin{tabular}{|c|c|c|c|c|c|c|c|}
\hline \multirow[b]{2}{*}{ Element } & \multirow{2}{*}{$\begin{array}{l}\text { Initial } \\
\text { Amount }\end{array}$} & FFTF & \multicolumn{3}{|c|}{ HFIR } & \multicolumn{2}{|c|}{ First Wall } \\
\hline & & 2909 & PTP & RBd & RB4(HF) & STARFIRE & MARS \\
\hline C & 1.000 & -0.03 & -0.02 & -0.00 & -0.00 & -1.33 & -1.35 \\
\hline
\end{tabular}


TABLE 9 (Cont'd)

New Elements (parts per thousand)

\begin{tabular}{|c|c|c|c|c|c|c|}
\hline \multirow[b]{2}{*}{ Element } & FFTF & \multicolumn{3}{|c|}{ HFIR } & \multicolumn{2}{|c|}{ First Wall } \\
\hline & $2101 \quad 2909$ & PTP & RB4 & RB4(HF) & STARFIRE & NARSS \\
\hline $\begin{array}{l}\mathrm{H} \\
\mathrm{He} \\
\mathrm{Be}\end{array}$ & $\begin{array}{l}\text { not calculated } \\
\text { not calculated } \\
0.212\end{array}$ & 197 & 0.039 & 0.037 & 85 & 389 \\
\hline
\end{tabular}

TABLE 10

TRANSMUTATIONS IN AI AFTER 5 VEARS IN 7 FUSION TEST FACILITIES

Fractional Change (\%) in Initial Constituents

Element

A1

$\begin{array}{r}\text { Initial } \\ \text { Amount } \\ \hline\end{array}$

FFTF

1.000

$-0.00$

\begin{tabular}{lll} 
PTP & $\frac{H F I R}{R B 4}$ & RB4(HF) \\
\hline-8.4 & -2.81 & -0.39
\end{tabular}

New Elements (parts per thousand)

Element

H

He

$\mathrm{Ne}$

$\mathrm{Na}$

$\mathrm{Mg}$

in

\begin{tabular}{ll}
\multicolumn{2}{c}{ FFTF } \\
\hline-2101 & -2909 \\
0.68 & 0.001 \\
0.139 & \\
0.001 & \\
0.171 & \\
2.77 & 0.042
\end{tabular}

\begin{tabular}{lll} 
& \multicolumn{1}{c}{ HFIR } & \\
\hline PTP & RB4 & RB4(HF) \\
\cline { 4 - 4 } 7.4 & 0.139 & 0.130 \\
0.120 & 0.024 & 0.024 \\
& & \\
0.137 & 0.029 & 0.028 \\
84.0 & 28.1 & 3.9
\end{tabular}

TABLE 11

TRANSMUTATIONS IN Si AFTER 5 YEARS IN 7 FUSION TEST FACILITIES

Fractional Change (\%) in Initial Constituents

\begin{tabular}{|c|c|c|c|c|c|c|}
\hline \multirow[b]{2}{*}{ Element } & \multirow{2}{*}{$\begin{array}{r}\text { Initial } \\
\text { Amount } \\
\end{array}$} & \multicolumn{2}{|c|}{ FFTF } & \multicolumn{3}{|c|}{ HFIR } \\
\hline & & 2101 & 2909 & PTP & RB4 & RB4(HF) \\
\hline Si & 1.000 & -0.02 & -0.01 & -0.14 & -0.04 & -0.01 \\
\hline
\end{tabular}

\begin{tabular}{l} 
Element \\
\hline $\mathrm{H}$ \\
$\mathrm{He}$ \\
$\mathrm{Na}$ \\
$\mathrm{Mg}$ \\
$\mathrm{A}$ \\
$\mathrm{P}$ \\
$\mathrm{S}$
\end{tabular}

FI0I 2 FFIF

\begin{tabular}{l} 
HFIR \\
\hline PTP \\
\hline
\end{tabular}

not calculated separately not calculated separately
0.095
0.034
0.072
0.013

$\begin{array}{lll}0.092 & 0.017 & 0.016 \\ 0.016 & 0.005 & 0.004 \\ 1.25 & 0.41 & 0.061 \\ 0.044 & 0.005 & \end{array}$

First Wa11 STARFIRE

$-1.32$ MARS

First Wall

$\begin{array}{cc}\text { STARFIRE } & \text { MARS } \\ 8.2 & 8.2 \\ 5.7 & 5.7 \\ 0.012 & 0.012 \\ 0.099 & 0.100 \\ 11.8 & 11.9 \\ 1.26 & 0.56\end{array}$

First Wall

$\begin{array}{ll}\text { STARFIRE } & - \text { MARS } \\ -1.36 & -1.37\end{array}$

First Wall

STARFIRE MARS

$\begin{array}{ll}0.011 & 0.012 \\ 5.0 & 5.0 \\ 8.6 & 8.7 \\ 0.029 & 0.016\end{array}$


TABLE 12

TRANSMUTATIONS IN CU AFTER 5 YEARS IN 7 FUSION TEST FACILITIES

Fractional Change (\%) in Initial Constituents

\begin{tabular}{|c|c|c|c|c|c|c|c|}
\hline \multirow[b]{2}{*}{ Element } & \multirow{2}{*}{$\begin{array}{r}\text { Initial } \\
\text { Amount }\end{array}$} & FFTF & \multicolumn{3}{|c|}{ HFIR } & \multicolumn{2}{|c|}{ First Wall } \\
\hline & & 2909 & PTP & RB4 & RB4(HF) & STARFIRE & MARS \\
\hline Cu & 1.000 & -1.38 & -43.0 & -23.7 & -4.2 & -5.8 & -4.1 \\
\hline
\end{tabular}

New Elements (parts per thousand)

Element

H

He

$\mathrm{Fe}$

Co

$\mathrm{Ni}$

2n

Ga

Ge

$\mathrm{Nb}$

H

He

Sr

Y

Ir

Mo

Tc

$\mathrm{Ru}$

$\mathrm{Rh}$

FFTF

not separately calculated not separately calculated

$\begin{array}{lr}0.088 & \\ 29.4 & 10.2 \\ 10.3 & 3.6\end{array}$

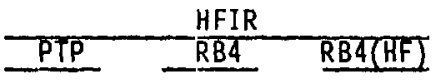

$\begin{array}{ccc}0.247 & 0.011 & 0.017 \\ 251.0 & 173.0 & 32.0 \\ 169.0 & 64.0 & 9.8 \\ 6.1 & 0.225 & \\ 3.3 & 0.038 & \end{array}$

TABLE 13

TRANSMUTATIONS IN ND AFTER 5 YEARS IN 7 FUSION TEST FACILITIES

$$
\frac{\begin{array}{r}
\text { Initial } \\
\text { Amount }
\end{array}}{1.000}
$$

\begin{tabular}{|c|c|}
\hline & \\
\hline 2101 & 2909 \\
\hline
\end{tabular}

Fractional Change

\begin{tabular}{|c|c|c|}
\hline & HFIR & \\
\hline PTP & $\mathrm{RB4}$ & RB4(HF) \\
\hline & & \\
\hline
\end{tabular}

$(\%)$

New Elements (parts per thousand)

\begin{tabular}{cc}
\multicolumn{2}{c}{ FFTF } \\
\hline-2101 & -2909 \\
0.109 & \\
0.034 & \\
& \\
0.005 & \\
0.236 & \\
106.0 & 40.0 \\
0.001 &
\end{tabular}

\begin{tabular}{ccc} 
& HFIR & \\
\hline PTP & RB4 & RB4(HF) \\
\cline { 3 - 4 } 0.236 & 0.022 & 0.005 \\
& 0.005 & 0.005 \\
& & \\
0.002 & 0.001 & 0.001 \\
0.126 & 0.037 & 0.040 \\
660.0 & 298.0 & 35.0 \\
1.48 & 0.020 & \\
0.151 & 0.001 & \\
0.001 & &
\end{tabular}

TABLE 14
First Wall STARFIRE MARS

$\begin{array}{cc}0.022 & 0.024 \\ 2.25 & 2.35 \\ 50.0 & 37.0 \\ 6.2 & 2.04\end{array}$

First Wall $\frac{\text { STARFIRE }}{-9.2}-\frac{\text { MARS }}{-5.3}$

First Wall STARFIRE MARS

$5.5 \quad 5.5$

$1.15 \quad 1.22$

$0.001 \quad 0.013$

$0.261 \quad 0.293$

$26.5 \quad 28.8$

$\begin{array}{ll}65.0 & 24.4\end{array}$

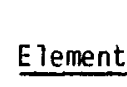

Mo $\frac{\begin{array}{r}\text { Initial } \\ \text { Amount }\end{array}}{1.000}$
Fractional Change

(\%) in Initial Constituents

$\begin{array}{ll}\frac{\text { FFTF }}{2101} & -2909 \\ -3.5 & -0.93\end{array}$

First Wall

$\begin{array}{ll}\text { STARFIRE } & - \text { MARS } \\ -2.44 & -1.81\end{array}$


TABLE 14 (Cont'd)

New Elements (parts per thousand)

\begin{tabular}{l} 
Element \\
\hline$H$ \\
$H \mathrm{He}$ \\
$\mathrm{Sr}$ \\
$\mathrm{Y}$ \\
$\mathrm{Zr}$ \\
$\mathrm{Nb}$ \\
$\mathrm{TC}$ \\
$\mathrm{Ru}$ \\
$\mathrm{Rh}$ \\
$\mathrm{Pd}$
\end{tabular}

$\frac{\text { FFTF }}{2101 \quad-2909}$

\begin{tabular}{c} 
HFIR \\
\hline PTP \\
- RBA
\end{tabular}

First Wall

not calculated separately

not calculated separately

$\begin{array}{llccl}0.001 & & & & \\ 0.029 & & 0.020 & 0.004 & 0.005 \\ 0.039 & & 0.038 & 0.010 & 0.009 \\ 22.5 & 6.72 & 38.0 & 17.2 & 6.3 \\ 11.7 & 2.56 & 24.6 & 8.0 & 1.81 \\ 0.109 & 0.003 & 1.22 & 0.162 & 0.003 \\ 0.009 & & 0.046 & 0.019 & \end{array}$

$\begin{array}{ll}0.109 & 0.113 \\ 0.31 & 0.32 \\ 1.71 & 1.62 \\ 4.3 & 5.0 \\ 14.0 & 9.3 \\ 4.0 & 1.78 \\ 0.009 & 0.001\end{array}$

TABLE 15

TRANSMUTATIONS IN W AFTER 5 YEARS IN 7 FUSION TEST FACILITIES

Fractional Change (\%) in Initial Constituents

\begin{tabular}{|c|c|c|c|c|c|c|}
\hline & Initial & \multicolumn{2}{|c|}{ FFTF } & \multicolumn{3}{|c|}{ HFIR } \\
\hline Element & Amount & 2101 & 2909 & PTP & RB4 & RB4 (HF) \\
\hline$W$ & 1.000 & -17.2 & -29.0 & -90.0 & -57.0 & -34.0 \\
\hline
\end{tabular}

\begin{tabular}{l} 
Element \\
\hline $\mathrm{H}$ \\
$\mathrm{He}$ \\
$\mathrm{Hf}$ \\
$\mathrm{Ta}$ \\
$\mathrm{Re}$ \\
$\mathrm{OS}$ \\
$\mathrm{Ir}$ \\
$\mathrm{Pt}$ \\
$\mathrm{Au}$ \\
$\mathrm{Hg}$ \\
$\mathrm{Tl}$ \\
$\mathrm{Pb}$
\end{tabular}

\begin{tabular}{cc}
\multicolumn{2}{c}{ FFTF } \\
\hline 2101 & -2909 \\
0.003 & \\
0.002 & \\
0.002 & \\
0.41 & 0.54 \\
117.0 & 155.0 \\
55.0 & 122.0 \\
0.080 & 10.8 \\
0.005 & 1.29
\end{tabular}

\begin{tabular}{ccc}
\multicolumn{3}{c}{ HFIR } \\
\hline PTP & RB4 & RB4(HF) \\
\cline { 3 - 4 } 0.001 & & \\
& & \\
0.001 & 0.008 & 0.47 \\
360.0 & 155.0 & 74.0 \\
66.0 & 80.0 & 128.0 \\
& 185.0 & 111.0 \\
44.0 & 74.0 & 30.0 \\
& 4.2 & 0.263 \\
185.0 & 72.0 & 0.086 \\
207.0 & 2.31 & \\
34.0 & 0.058 &
\end{tabular}

First Wall

\begin{tabular}{cc}
\multicolumn{2}{c}{ First Wall } \\
STARFIRE & MARS \\
-36.0 & -10.0
\end{tabular}

First Wall STARFIRE MARS

$\begin{array}{cc}0.218 & 0.254 \\ 0.213 & 0.226 \\ 0.211 & 0.39 \\ 1.69 & 22.0 \\ 140.0 & 72.0 \\ 161.0 & 5.2 \\ 44.0 & \\ 10.3 & \\ 0.012 & \\ 0.007 & \end{array}$

